\title{
Microwave-assisted urea catalyzed Knoevenagel condensation of aldehydes with active methylene compounds
}

\author{
W. T. Purba, P. S. Roy, S. Jannat, S. A. Begum and M. M. Rahman* \\ Department of Chemistry, Shahjalal University of Science \& Technology, Sylhet 3114, Bangladesh
}

Received: 29 September 2019

Revised: 27 October 2019

Accepted: 11 Nobember 2019

DOI: https://doi.org/10.3329/bjsir.v55i2.47637

\begin{abstract}
Rapid and efficient method for the synthesis of substituted olefins such as 2-(4-chlorophenylmethylene) malononitrile, 2-(4-hydroxyphenylmethylene) malononitrile and 2-cyano-3-(4-hydroxyphenyl) acrylamide etc under the influence of microwave irradiation are described. Urea has been utilized as an efficient catalyst for the Knoevenagel condensation of aldehydes with acidic active methylene compounds such as malononitrile, ethylcyanoacetate and cyanoacetamide to afford substituted olefins under the influence of microwave irradiation. The reaction proceeds smoothly under mild and solvent free conditions and the products are obtained in good yield. The method is applicable for a wide range of aldehydes including aromatic and heterocyclic substrates.
\end{abstract}

Keywards: Microwave; Active methylene; Green procedure; Urea catalyst; Substituted cyanoacetamide; Knoevenagel condesation

\section{Introduction}

The Knoevenagel condensation has numerous application in the synthesis of fine chemicals (Freeman, 1981), hetero Diels-Alder reactions (Borah et al., 2005) as well as heterocyclic compounds of biological significance (Tietze, 1996). The condensation of aldehydes with active methylene compounds is an important and widely employed method for carbon-carbon double bond formation in organic synthesis. The reaction is usually carried out by bases (Yang et al., 2006), such as sodium hydroxide or sodium ethoxide in organic solvents. Organic weak bases such as aliphatic amines, ethylenediamine and piperidine or corresponding ammonium salt are also used for Knoevenagel reaction (Acker et al., 1962). The Knoevenagel condensations between aldehydes and malononitrile in dry media catalyzed by $\mathrm{ZnCl}_{2}$ (Rao, 1991), silica gel (Cruz et al., 1996) have been reported.

Bhuiyan et al. (2012) reported the synthesis of arylidene malononitriles by the reaction of malononitriles and aldehydes.

Microwave-assisted solvent-free reactions (Wang, 2001) represent very powerful green chemical technology procedures from both the economical and synthetic point of view. In literature report the synthesis of substituted olefins have been described by Sun Qi et al. (2005) using conventional thermal methods involved long reaction time.

The ability of microwave irradiation to reduce reaction time led to an idea for this synthesis to be carried out under the influence of microwave irradiation. In our previous report we have reported microwave assisted synthesis of arylidene derivatives using $\mathrm{NH}_{4} \mathrm{OAc}$ as a catalyst (Ahmed et al., 2018). In continuation of our interest to study the organic reaction or transformation under microwave irradiation we herein, report synthesis of known and new aryledene malononitriles, acrylamide using microwave irradiation.

\section{Materials and methods}

All chemicals were purchased from (Sigma-Aldrich, Merck, and Alfa Aesar) and were used without further 
purification. The Chemicals 4-fluorobenzaldehyde (98\%), 4-hydroxybenzaldehyde (98\%), 4-nitrobenzaldehyde (98\%), 2-nitrobenzaldehyde (98\%), 3-nitrobenzaldehyde (98\%), 4-chlorobenzaldehyde (97\%) and cyanoacetamide (98\%) from Sigma-Aldrich, Cinnamaldehyde (98\%), and ethylcyanoacetate from Merck, 2-furaldehyde (98\%), 4-methylbenzaldehyde (98\%), 4-methoxybenzaldehyde (99\%) and malononitrile (99\%) were purchased from Alfa Aser. GALLEN KAMP melting point apparatus was used to record the melting point of the solid compound, the heating was done carefully to ensure a steady rise of temperature. ${ }^{1} \mathrm{H}$ NMR spectra were recorded on a JEOL JNM ECS FT NMR at $400 \mathrm{MHz}$ spectrometer using tetramethylsilane as an internal standard. Coupling constants $J$ are given in $\mathrm{Hz}$. Infrared spectra were observed on a Shimadzu FTIR- spectrometer (IR prestige 21). Analytical TLC was performed on pre-coated alumina sheet and the product was visualized by UV light. All reactions were carried out in a domestic microwave oven (Samsung, MW76ND).

\section{General procedure}

To a mixture of aromatic aldehyde $1(2 \mathrm{mmol})$ and active methylene compound 2 (2 mmol) was added catalytic amount of urea( $10 \mathrm{~mol} \%, 0.2 \mathrm{mmol})$ in a Pyrex glass 50 $\mathrm{mL}$ beaker and mixed thoroughly using a glass rod. The mixture was then subjected to microwave irradiation at $180 \mathrm{~W}-800 \mathrm{~W}$ for 1 to $5 \mathrm{~min}$. The progress of the reaction was followed by TLC ( $n$-hexane: ethyl acetate). After complete conversion and cooling of the reaction, the solid reaction mixture was washed with water to remove urea. Recrystallization of the mixture was done using ethyl acetate and $n$-hexane solvent mixture to give the pure product 3 under the scheme 1 . 2-(4-nitrophenylmethylene)malononitrile (3a): yield 90\%, yellowish white solid, m.p. $158-159^{\circ} \mathrm{C}$, (lit. $160^{\circ} \mathrm{C}$ (Sun Qi et al 2005)); IR (KBr): v $v_{\max }$ : 3039(sp $\left.{ }^{2} \mathrm{C}-\mathrm{H}\right), 2231(\mathrm{C} \equiv \mathrm{N}), 1604$ $(\mathrm{C}=\mathrm{C})$ and 1521 and $1344(\mathrm{~N}-\mathrm{O}) \mathrm{cm}^{-1} ;{ }^{1} \mathrm{H} \mathrm{NMR}(400 \mathrm{MHz}$, $\left.C D \mathrm{Cl}_{3}\right) \delta(\mathrm{ppm}): 8.41(2 \mathrm{H}, \mathrm{d}, J=8.8 \mathrm{~Hz},=\mathrm{CH}), 8.09(2 \mathrm{H}, \mathrm{d}, J$ $=8.8 \mathrm{~Hz}, \mathrm{Ar}), 7.89(1 \mathrm{H}, \mathrm{s},-\mathrm{CH})$.

2-(4-methylbenzylidene)malononitrile (3b): yield $96 \%$, white solid, m.p. $131-132^{\circ} \mathrm{C}$, (lit. $134^{\circ} \mathrm{C}$ (Ren et al 2002)); IR $(\mathrm{KBr}): v_{\max }\left(\mathrm{cm}^{-1}\right): 3035\left(\mathrm{sp}^{2} \mathrm{C}-\mathrm{H}\right), 2927\left(\mathrm{sp}^{3} \mathrm{C}-\mathrm{H}\right), 2223(\mathrm{C} \equiv$ $\mathrm{N})$ and $1589(\mathrm{C}=\mathrm{C}) \mathrm{cm}^{-1}$; ${ }^{1} \mathrm{H} \mathrm{NMR}\left(400 \mathrm{MHz}, \mathrm{CDCl}_{3}\right)$ $\delta(\mathrm{ppm}): 7.80(2 \mathrm{H}, \mathrm{d}, J=8.2 \mathrm{~Hz}, \mathrm{Ar}), 7.71(1 \mathrm{H}, \mathrm{s},=\mathrm{CH}), 7.32$ (2H, d, J=8.2 Hz, Ar.), 2.44 (3H, s, $\left.-\mathrm{CH}_{3}\right)$;

2-(4-fluorobenzylidene)malononitrile (3c): yield 94\%, light brown solid m.p. $119^{\circ} \mathrm{C}$, (lit.124-125 ${ }^{\circ} \mathrm{C}$ (Fan et al 2004); IR $(\mathrm{KBr}): \mathrm{v}_{\max }\left(\mathrm{cm}^{-1}\right): 3043\left(\mathrm{sp}^{2} \mathrm{C}-\mathrm{H}\right), 2231(\mathrm{C} \equiv \mathrm{N})$ and 1658 $(\mathrm{C}=\mathrm{C}) \mathrm{cm}^{-1} ;{ }^{1} H$ NMR $(400 \mathrm{MHz}, D M S O) \delta(\mathrm{ppm}): 8.53(1 \mathrm{H}, \mathrm{s}$, $=\mathrm{CH}), 8.02-8.06(2 \mathrm{H}, \mathrm{t}, J=8 \mathrm{~Hz}, \mathrm{Ar}), 7.48-7.52(2 \mathrm{H}, \mathrm{m}, \mathrm{Ar})$.

2-(4-Chlorophenylmethylene)malononitrile (3d): yield $95 \%$, white solid, m.p. $158-160^{\circ} \mathrm{C}$, (lit. $165^{\circ} \mathrm{C}$ (Sun Qi et al 2005); IR (KBr): $v_{\max }\left(\mathrm{cm}^{-1}\right): 3034\left(\mathrm{sp}^{2} \mathrm{C}-\mathrm{H}\right), 2227(\mathrm{C} \equiv \mathrm{N})$ and $1585(\mathrm{C}=\mathrm{C}) \mathrm{cm}^{-1} ;{ }^{1} \mathrm{HNMR}\left(400 \mathrm{MHz}, \mathrm{CDCl}_{3}\right) \delta(\mathrm{ppm})$ : 7.72(1H, s, $=\mathrm{CH}), 7.83(2 \mathrm{H}, \mathrm{d}, J=8 \mathrm{~Hz}, \mathrm{Ar}), 7.53(2 \mathrm{H}$, $\mathrm{d}, J=8 \mathrm{~Hz}, \mathrm{Ar})$.

2-(3-nitrophenylmethylene)malononitrile (3e): yield $89 \%$, creamy white solid, m.p. $102-103^{\circ} \mathrm{C}$, (lit. $104-105^{\circ} \mathrm{C}$ ( Sun Qi et al 2005); IR (KBr): $v_{\max }\left(\mathrm{cm}^{-1}\right): 3086\left(\mathrm{sp}^{2} \mathrm{C}-\mathrm{H}\right), 2225(\mathrm{C} \equiv$ $\mathrm{N}), 1597(\mathrm{C}=\mathrm{C})$ and $1529 \& 1355(\mathrm{~N}-\mathrm{O}) \mathrm{cm}^{-1} ;{ }^{1} \mathrm{H}$ NMR $(400$ $\left.\mathrm{MHz}, \mathrm{CDCl}_{3}\right) \delta(\mathrm{ppm}): 8.67(1 \mathrm{H}, \mathrm{t}, J=1.8 \mathrm{~Hz}, \mathrm{Ar}), 8.49(1 \mathrm{H}$, $\mathrm{dd}, J=1.5,8.1 \mathrm{~Hz}$, Ar), $8.34(1 \mathrm{H}, \mathrm{d}, J=7.8 \mathrm{~Hz}$, Ar $), 7.90$ $(\mathrm{s}, 1 \mathrm{H},=\mathrm{CH}), 7.80(1 \mathrm{H}, \mathrm{t}, J=8.1 \mathrm{~Hz}, \mathrm{Ar})$.
Scheme 1

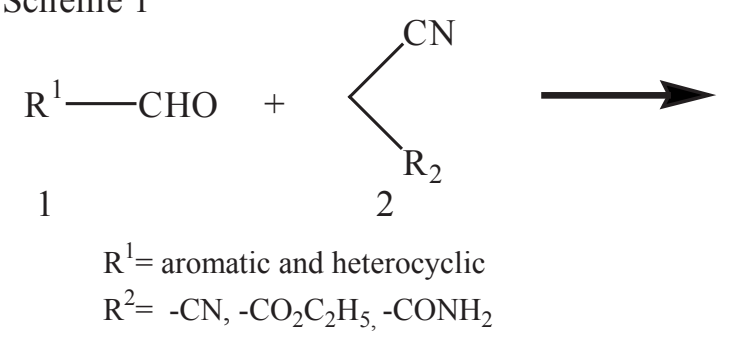

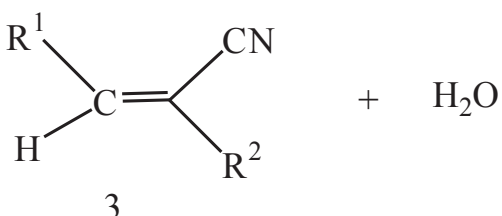


2-(4-Hydroxyphenylmethylene)malononitrile (3f): yield $96 \%$, yellow solid, m.p. $171-172^{\circ} \mathrm{C}$, (lit. $189-190^{\circ} \mathrm{C}$ ( Sun Qi et al 2005$)$; IR (KBr): $v_{\text {max }}\left(\mathrm{cm}^{-1}\right): \quad 3354(-\mathrm{OH})$, $3030\left(\mathrm{sp}^{2} \mathrm{C}-\mathrm{H}\right), 2227(\mathrm{C} \equiv \mathrm{N})$, and $1670(\mathrm{C}=\mathrm{C}) \mathrm{cm}^{-1} ;{ }^{1} H$ NMR (400 $\left.\mathrm{MHz}, \mathrm{CDCl}_{3}\right) \delta(\mathrm{ppm}): 7.89(2 \mathrm{H}, \mathrm{d}, J=9.0 \mathrm{~Hz}$, Ar), $7.65(1 \mathrm{H}, \mathrm{s},=\mathrm{CH}), 6.97(2 \mathrm{H}, \mathrm{d}, J=9.0 \mathrm{~Hz}, \mathrm{Ar})$, $5.75(1 \mathrm{H}, \mathrm{s}, \mathrm{OH})$.

2-(2-Furylmethylene)malononitrile $\quad(3 \mathrm{~g})$ : yield $90 \%$, blackish brown solid, m.p. $62-63^{\circ} \mathrm{C}$, (lit. $72^{\circ} \mathrm{C}$ ( Sun Qi et al 2005 ); IR (KBr): $v_{\max }\left(\mathrm{cm}^{-1}\right): 3124\left(\mathrm{sp}^{2} \mathrm{C}-\mathrm{H}\right), 2223(\mathrm{C} \equiv$ N) $1606(\mathrm{C}=\mathrm{C})$ and $1151(\mathrm{C}-\mathrm{O}-\mathrm{C}) \mathrm{cm}^{-1} ;{ }^{1} \mathrm{H} \mathrm{NMR}(400$ $\left.\mathrm{MHz}, \mathrm{CDCl}_{3}\right) \delta(\mathrm{ppm}): 7.80(1 \mathrm{H}, \mathrm{d}, \mathrm{J}=1.6 \mathrm{~Hz}, \mathrm{Ar}), 7.50$ $(1 \mathrm{H}, \mathrm{s},=\mathrm{CH}) ; 6.70(1 \mathrm{H}, \mathrm{dd}, J=3.6 \& 1.6 \mathrm{~Hz}, \mathrm{Ar}), 7.35$ $(1 \mathrm{H}, \mathrm{d}, \mathrm{J}=4.0 \mathrm{~Hz}, \mathrm{Ar})$.

2-(3-pyridylmethylene)malononitrile (3h): yield 95\%, brownish solid, m.p. $80-82^{\circ} \mathrm{C}$, (lit. $83-84{ }^{\circ} \mathrm{C}$ ( SunQi et al 2005 ); IR (KBr): $v_{\text {max }}\left(\mathrm{cm}^{-1}\right): 3032\left(\mathrm{sp}^{2} \mathrm{C}-\mathrm{H}\right), 2227(\mathrm{C} \equiv \mathrm{N})$ $1589(\mathrm{C}=\mathrm{C}) ;{ }^{1} H$ NMR (400 $\left.\mathrm{MHz} \mathrm{CDCl}_{3}\right) \delta(\mathrm{ppm}): 8.89$ $(1 \mathrm{H}, \mathrm{d}, J=2.4 \mathrm{~Hz}, \mathrm{Ar}), 8.83(1 \mathrm{H}, \mathrm{dd}, J=1.2 \& 4.8 \mathrm{~Hz}$, Ar), $8.50(1 \mathrm{H}, \mathrm{dd}, \mathrm{J}=1.5,8.0 \mathrm{~Hz}, \operatorname{Ar}) .7 .83(1 \mathrm{H}, \mathrm{s},=\mathrm{CH})$, 7.53(1H, dd, J = 4.8, 7.8 Hz, Ar).

Ethyl 2-cyano-3-styryl-2-propenoate (3i): yield 92\%, yellowish solid m.p. $111-112^{\circ} \mathrm{C}$, (lit. $115^{\circ} \mathrm{C}(\mathrm{Sun}$ Qi et al 2005); IR (KBr): $v_{\text {max }}\left(\mathrm{cm}^{-1}\right): 3030\left(\mathrm{sp}^{2} \mathrm{C}-\mathrm{H}\right), 2250(\mathrm{C} \equiv \mathrm{N})$, 1743 $(\mathrm{C}=\mathrm{O})$ and $1662(\mathrm{C}=\mathrm{C}) \mathrm{cm}^{-1} ;{ }^{1} \mathrm{H} \mathrm{NMR}(400 \mathrm{MHz}$, $\left.C D C l_{3}\right) \delta(\mathrm{ppm}): 8.00(1 \mathrm{H}, \mathrm{dd}, J=3.9 \& 6.9 \mathrm{~Hz},=\mathrm{CH})$ 7.57- 7.61 (2H, m, Ar), 7.41- 7.44(3H, m, Ar), $7.26(2 \mathrm{H}$, $\mathrm{q}, J=3.6 \mathrm{~Hz},=\mathrm{CH}.), 4.35\left(2 \mathrm{H}, \mathrm{q}, J=7.2 \mathrm{~Hz}, \mathrm{OCH}_{2}\right), 1.38$ $\left(3 \mathrm{H}, \mathrm{t}, J=7.2 \mathrm{~Hz},-\mathrm{CH}_{3}\right)$.

2-cyano-3-(4-hydroxyphenyl)acrylamide (3j): yield $95 \%$, white solid, m.p. $245-246^{\circ} \mathrm{C}$, (lit. $245^{\circ} \mathrm{C}$ (Sun Qi et al 2005); IR (KBr): $v_{\max }\left(\mathrm{cm}^{-1}\right): 3454$ and 3366 (N-H str. For $\mathrm{NH}_{2}$ ), 3186 (O-H str.), 3079 (aromatic C-H str.), 3030 (sp2 C-H str.), 2229 (C $\equiv \mathrm{N}$ str.), 1658 (CO str.), 1624 (alkene $\mathrm{C}=\mathrm{C}$ str.), 1600 and 1514 (aromatic $\mathrm{C}=\mathrm{C}$ str.), 1290 (C-N str), 1251 (C-O str). ${ }^{1} \mathrm{H} \mathrm{NMR}$ (400 MHz, $\left.\mathrm{CDCl}_{3}\right) \delta(\mathrm{ppm}): 10.53(\mathrm{br}, \mathrm{s}, 1 \mathrm{H}), 8.05(\mathrm{~s}, 1 \mathrm{H}), 7.87(\mathrm{~d}$, $J=8.8,2 \mathrm{H}), 7.74(\mathrm{~s}, 1 \mathrm{H}, \mathrm{NH}), 7.61(\mathrm{~s}, 1 \mathrm{H}, \mathrm{NH}), 6.93(\mathrm{~d}$, $J=8.8,2 \mathrm{H})$.

2-cyano-3-(4-fluorophenyl)acrylamide (3k): yield $96 \%$, white solid, m.p. $151^{\circ} \mathrm{C} ; I R(K B r): v_{\max }\left(\mathrm{cm}^{-1}\right)$ : 3488and 3381 (N-H str. For $\mathrm{NH}_{2}$ ), 3169 (aromatic C-H str.), 3024 (sp2 C-H str.), 2216 (C $\equiv \mathrm{N}$ str.), 1701 (CO str.), 1687 (alkene $\mathrm{C}=\mathrm{C}$ str.), 1600 and1504 (aromatic $\mathrm{C}=\mathrm{C}$ str.), 1386 (C-N str), 1233 (C-O str.); ${ }^{1} \mathrm{H}$ NMR (400 MHz,
$\left.C D \mathrm{Cl}_{3}\right) \delta(\mathrm{ppm}): 8.32(\mathrm{~s}, 1 \mathrm{H}), 8.01(\mathrm{~d}, J=8.4,2 \mathrm{H}), 7.22(\mathrm{~d}$, $J=8.4,2 \mathrm{H}), 6.38(\mathrm{~s}, 1 \mathrm{H}, \mathrm{N}-\mathrm{H}), 6.11(\mathrm{~s}, 1 \mathrm{H}, \mathrm{N}-\mathrm{H})$.

2-cyano-3-(4-nitrophenyl)acrylamide (31): yield $85 \%$, light brown solid, m.p. $229-230^{\circ} \mathrm{C}$, ; IR $(\mathrm{KBr}): v_{\max }\left(\mathrm{cm}^{-1}\right)$ : 3442 and 3346 (N-H str., $\mathrm{NH}_{2}$ ), 3196 (aromatic C-H str.), 3024 (sp2 C-H str.), 2224 (C $\equiv \mathrm{N}$ str.), 1695 (CO str.), 1622 (alkene $\mathrm{C}=\mathrm{C}$ str.), 1600 and1494 (aromatic $\mathrm{C}=\mathrm{C}$ str.), 1510 and 1346 (nitro $\mathrm{N}=\mathrm{O}$ str.), 1327 (C-N str), 1203 (C-O str.), ${ }^{1} \mathrm{H} N M R$ (400 $\left.\mathrm{MHz}, C D C l_{3}\right) \delta(\mathrm{ppm})$ : $8.39(\mathrm{~d}, J=8.8 \mathrm{~Hz}, 2 \mathrm{H}), 8.30(\mathrm{~s}, 1 \mathrm{H}), 8.13(\mathrm{~d}, J=8.8,2 \mathrm{H})$, 8.05(s, 1H, N-H), 7.91(s, 1H, N-H).

2-cyano-3-(furan-2-yl)acrylamide $(3 \mathrm{~m})$ : yield $88 \%$, light brown solid, m.p. $93-94^{\circ} \mathrm{C} ; \mathrm{IR}(\mathrm{KBr}): \mathrm{v}_{\max }\left(\mathrm{cm}^{-1}\right)$ : 3488and 3381 (N-H str. For $\mathrm{NH}_{2}$ ), 3169 (aromatic C-H str.), 3024 (sp2 C-H str.), 2216 (C $\equiv \mathrm{N}$ str.), 1701 (CO str.), 1687 (alkene $\mathrm{C}=\mathrm{C}$ str.), 1600 and 1504 (aromatic $\mathrm{C}=\mathrm{C}$ str.), 1386 (C-N str), 1233 (C-O str.). ${ }^{1} \mathrm{H} \mathrm{NMR} \mathrm{(400} \mathrm{MHz,}$ $\left.C D l_{3}\right) \delta(\mathrm{ppm}): 8.10(\mathrm{~s}, 1 \mathrm{H}), 7.76(\mathrm{~d}, J=1.6,1 \mathrm{H}), 7.25(\mathrm{~d}$, $J=3.6,1 \mathrm{H}), 6.66(\mathrm{dd}, J=3.6, J=1.6,1 \mathrm{H}), 6.29(\mathrm{~s}, 1 \mathrm{H}$, N-H), 5.76(s, 1H, N-H).

\section{Results and discussion}

In this report, we highlight our findings on the microwave assisted urea catalysed condensation of active methylene compounds, such as malononitrile, ethylcyanoacetate and cyanoacetamide with aromatic and heterocyclic aldehydes. Initially, the condensation reaction of $p$-hydroxybenzaldehyde with malononitrile and $p$-hydroxybenzaldehyde with cyanoacetamide was carried out at $300 \mathrm{~W}$ for 1-3 min without urea. In both the cases the reactions were not proceeded. This is worth to mention that in every case we obtained the starting materials only. However, when the reaction was conducted using urea as catalyst, at $300 \mathrm{~W} / 1-3 \mathrm{~min}$, the desired product is obtained. Accordingly, treatment of para-nitrobenzaldehyde with malononitrile in the presence $10 \mathrm{~mol} \%$ of urea catalyst at $300 \mathrm{~W} / 1-3 \mathrm{~min}$ resulted in the formation of 3 a $90 \%$ yield under solvent free conditions. In a similar manner, a wide range of substrates such as 4-methyl, 4-fluoro, 4-chloro, 3-nitro, 4-hydroxy aldehydes etc with active methylene malononitrile including heterocyclic aldehydes 2-furalaldehyde and pyridine-4-carboxaldehyde react efficiently under the same conditions (Table 1) to give the corresponding olefins 3b, 3c, 3d, 3e, and $3 f$ respectively. 
Table I. Products of urea catalyzed microwave-assisted Knoevenagel condensation of aldehydes with active methylene compounds

\begin{tabular}{|c|c|c|c|c|c|c|}
\hline Entry & $\mathrm{R}^{1}$ & $\mathrm{R}^{2}$ & Reaction time and Watt & Products $^{\mathrm{a}}$ & Melting point ${ }^{\mathrm{C}}\left({ }^{\circ} \mathrm{C}\right)$ & Yield $^{\mathrm{b}}(\%)$ \\
\hline i & $\mathrm{p}-\mathrm{NO}_{2} \mathrm{C}_{6} \mathrm{H}_{5}$ & $-\mathrm{CN}$ & $1 \mathrm{~min} / 300 \mathrm{~W}$ & $3 a$ & $\begin{array}{c}158-159 \\
\left(160{ }^{\circ} \mathrm{C}\right)\end{array}$ & 90 \\
\hline ii & $\mathrm{p}-\mathrm{CH}_{3} \mathrm{C}_{6} \mathrm{H}_{5}$ & $-\mathrm{CN}$ & $1.5 \mathrm{~min} / 300 \mathrm{~W}$ & $3 b$ & $\begin{array}{c}131-132 \\
\left(134{ }^{\circ} \mathrm{C}\right)\end{array}$ & 96 \\
\hline iii & $\mathrm{p}-\mathrm{FC}_{6} \mathrm{H}_{5}$ & $-\mathrm{CN}$ & $3.5 \mathrm{~min} / 300 \mathrm{~W}$ & $3 \mathrm{c}$ & $\begin{array}{c}119 \\
\left(124-125^{\circ} \mathrm{C}\right)\end{array}$ & 94 \\
\hline iv & $\mathrm{p}-\mathrm{ClC}_{6} \mathrm{H}_{5}$ & $-\mathrm{CN}$ & $2.5 \mathrm{~min} / 450 \mathrm{~W}$ & $3 d$ & $\begin{array}{c}158-160 \\
\left(165^{\circ} \mathrm{C}\right)\end{array}$ & 95 \\
\hline $\mathrm{v}$ & $\mathrm{p}-\mathrm{NO}_{2} \mathrm{C}_{6} \mathrm{H}_{5}$ & $-\mathrm{CN}$ & $1.0 \mathrm{~min} / 180 \mathrm{~W}$ & $3 e$ & $\begin{array}{c}102-103 \\
\left(104-105^{\circ} \mathrm{C}\right)\end{array}$ & 89 \\
\hline vi & $\mathrm{p}-\mathrm{OHC}_{6} \mathrm{H}_{5}$ & $-\mathrm{CN}$ & $2 \mathrm{~min} / 180 \mathrm{~W}$ & $3 f$ & $\begin{array}{c}171-172 \\
\left(189-190{ }^{\circ} \mathrm{C}\right)\end{array}$ & 96 \\
\hline vii & 2-furyl & $-\mathrm{CN}$ & $2.5 \mathrm{~min} / 450 \mathrm{~W}$ & $3 g$ & $\begin{array}{l}62-63 \\
\left(72{ }^{\circ} \mathrm{C}\right)\end{array}$ & 90 \\
\hline viii & 3-pyridyl & $-\mathrm{CN}$ & $1.0 \mathrm{~min} / 180 \mathrm{~W}$ & $3 \mathrm{~h}$ & $\begin{array}{c}80-83 \\
\left(83-84^{\circ} \mathrm{C}\right)\end{array}$ & 95 \\
\hline ix & $\mathrm{PhCH}=\mathrm{CH}$ & $-\mathrm{CO}_{2} \mathrm{C}_{2} \mathrm{H}_{5}$ & $5 \mathrm{~min} / 300 \mathrm{~W}$ & $3 \mathrm{i}$ & $\begin{array}{l}111-112 \\
\left(115^{\circ} \mathrm{C}\right)\end{array}$ & 92 \\
\hline $\mathrm{x}$ & $\mathrm{p}-\mathrm{OHC}_{6} \mathrm{H}_{5}$ & $-\mathrm{CONH}_{2}$ & $2 \mathrm{~min} / 300 \mathrm{~W}$ & $3 \mathrm{j}$ & $\begin{array}{l}245-246 \\
\left(245^{\circ} \mathrm{C}\right)\end{array}$ & 95 \\
\hline $\mathrm{xi}$ & $\mathrm{p}-\mathrm{FC}_{6} \mathrm{H}_{5}$ & $-\mathrm{CONH}_{2}$ & $5 \mathrm{~min} / 300 \mathrm{~W}$ & $3 \mathrm{k}$ & $150-151$ & 96 \\
\hline xii & $\mathrm{p}-\mathrm{NO}_{2} \mathrm{C}_{6} \mathrm{H}_{5}$ & $-\mathrm{CONH}_{2}$ & $1.5 \mathrm{~min} / 300 \mathrm{~W}$ & 31 & $229-230$ & 85 \\
\hline xiii & 2-furyl & $-\mathrm{CONH}_{2}$ & $2 \mathrm{~min} / 450 \mathrm{~W}$ & $3 \mathrm{~m}$ & $93-94$ & 88 \\
\hline
\end{tabular}

${ }^{a}$ All products were characterized by m.p. and spectral techniques and melting was compared with literature value. ${ }^{\mathrm{b}}$ Isolated yield; ${ }^{\mathrm{c}}$ Values in parenthesis are lit M.P. 
Furthermore, the treatment of aldehyde such as cinnamaldehyde with ethylcyanoacetate also give olefinic compounds $3 \mathrm{i}$ under similar conditions (Table 1, Scheme 1). Both electron-rich and electron-deficient aldehydes gave high yield of products. Heterocyclic aldehydes such as 2-furaldehyde and pyridine-4-carboxaldehyde gave the olefinic derivatives 2-furylmethylene, $3 \mathrm{~g}$ and 2-(3-pyridylmethylene) olefinic $3 \mathrm{~h}$ derivatives at $90 \%$ and $95 \%$ yield respectively. Similar reaction of 4-hydroxy, 4-fluoro, 4-nitro, and 2-furylaldeydes with active methylene compounds 2-cyanoacetamide gave the corresponding olefins derivatives 2-cyano (3-(hydroxyphenyl)- propaneamide (3j), 2-cyano (3-(4-fluorophenyl)- propaneamide (3k), 2-cyano (3-(4-nitrophenyl)- propaneamide (31), and 2-cyano (3-(furylphenyl)- propaneamide $(3 \mathrm{~m})$ respectively. Nitro, hydroxyl, heterocyclic group are well tolerable due to the short reaction time and relatively mild reaction conditions. The reaction does not proceed under similar condition in the absence of urea catalyst. The reaction does not take place at all, only starting materials obtained in reaction mixture.

All the products were characterized by IR and ${ }^{1} \mathrm{H}$ NMR data analysis. The ${ }^{1} \mathrm{H}$ NMR spectra of the products $3 \mathrm{a}-3 \mathrm{i}$ showed the olefinic proton around $\delta 7-9 \mathrm{ppm}$ in $\mathrm{CDCl}_{3}$ as singlet peak. In the IR spectrum, the absorption band observed at $1705-1730 \mathrm{~cm}^{-1}$ is due to carbonyl, and the band at $2214-2225 \mathrm{~cm}^{-1}$ is due to the $-\mathrm{CN}$ groups and the band at $1650-1550 \mathrm{~cm}^{-1}$ is due to $\mathrm{C}=\mathrm{C}$ groups. The spectroscopic data of all the products are given sequentially.

\section{Conclusion}

In conclusion, we have synthesized several substituted olefins using urea as catalyst under solvent free condition. The use of urea as a catalyst helps to avoid the use of environmentally unfavorable organic solvents as reaction medium since the reaction proceeds smoothly under solvent free conditions. This method could be applied to a wide range of aldehydes including aromatic and heterocyclic substrates. A microwave-assisted solvent-free reaction conditions with short time and inexpensive and easily available catalyst are the key features involved in the present protocol.

\section{Acknowledgement}

The authors are grateful to the Department of Chemistry for giving the opportunity for experimental work. We gratefully acknowledge the University Research Centre, SUST for financial support.

\section{References}

Acker DS and Hertler WR, (1962), Substituted quinodimethans. I. Preparation and chemistry of 7, 7, 8, 8-tetracyanoquinodimethan. Journal of the American Chemical Society 84(17): 3370-3374. DOI: 10.1021/ja00876a028

Ahmed MR, Nasiruddin N, Alim MA, Jaman N, Islam MA, Begum SA, Rahman MM, Ahmed MF and Mannan MA (2018), Facile and rapid Synthesis of arylidene derivatives by microwave assisted $\mathrm{NH}_{4} \mathrm{OAc}$ catalyzed under solvent freecondition, Organic Chem: Curr Res pp 7-3.

Borah HN, Deb ML, Boruah RC and Bhuyan PJ (2005), Stereoselective intramolecular hetero Diels-Alder reactions of 1-oxa-1, 3-butadienes: synthesis of novel annelated pyrrolo [1, 2-a] indoles. Tetrahedron letters, 46(19): 3391-3393.

Bhuiyan MMH, Hossain MI, Alam MA and Mahmud MM (2012), Microwave assisted Knoevenagel condensation: Synthesis and antimicrobial activities of some arylidene-malononitriles, Chemistry Journal, 2(01): 31-37.

De la, Cruz P, Díez-Barra E, Loupy A and Langa F (1996), Silica gel catalysed Knoevenagel condensation in dry media under microwave irradiation. Tetrahedron letters 37(7): 1113-1116.

Freeman F (1981) An efficient synthesis of 2-thienyl methylene malononitrile, Chem. Rev. 80: 329-350.

Rao PS and Venkataratnam RV (1991), Zinc chloride as a new catalyst for Knoevenagel condensation. Tetrahedron letters 32(41): 5821-5822. DOI: 10.1016/S0040-4039(00)93564-0

Sun Qi, Shi, Lan-Xiang S, Ze-Mei G, Tie-Ming C and Run-Tao L (2005), An efficient and green procedure for the Knoevenagel condensation catalyzed by urea, Chinese Journal of Chemistry 23(6): 745-748. DO: 10.1002/cjoc. 200590745

Tietze LF (1996), Domino reactions in organic synthesis. Chemical reviews 96(1): 115-136. DOI: 10.1021/ cr950027e 
Yang Y, Dong ODW, Pan W, Zhang J and Liu Q (2006), Facile and clean synthesis of $\alpha$-alkenoyl ketene-(S,S)-acetal via the aldol condensation reactions in water, Tetrahedron 62: 10-111.
Wang C, Li Guishen S, Li G, Feng J, Li S and Xiaolu (2001), Synthesis of 5-alkylene barbituric acid in solventless under microwave irradiation, Chin. J. Org. Chem. 21(4): 310-312. 